\begin{tabular}{lllll} 
Abstract 178 & Table 2 & & \\
\hline $\begin{array}{l}\text { Cardiac Morphology } \\
\text { and Fibrosis }\end{array}$ & Control & Iso & \\
\cline { 2 - 5 } & & Immunocompetent & Immunocompromised & $\mathbf{P}$ \\
& & & $35 \pm 5$ & value \\
\hline Fibrotic Mass (mg) & 0 & $16 \pm 1$ & & 0.02 \\
\hline
\end{tabular}

Results ISO caused changes in: 1) cardiac function - increasing end diastolic and end systolic volumes while decreasing ejection fraction, 2) cardiac morphology - increasing the left ventricular mass resulting in hypertrophy (enlargement of the heart), and 3) cardiac fibrosis - apex of the heart being the most vulnerable. A differential-distribution of fibrosis-severity across the heart has also been detected.

Discussion In agreement with initial hypothesis, there was a differed-response between Immunocompetent and Immunocompromised subgroups: the Immunocompetent showed a trend towards cardiac hypertrophy, whereas the Immunocompromised were more vulnerable to fibrosis. This cardioprotective role of the immune system could be due to CXCR4 signalling that is also implicated in cancer-metastasis, stem-cellmigration, angiogenesis, and haematopoiesis, which we will follow up.

Conclusion ISO-murine-disease-model has been established and characterised to study myocardial damage, with cardiac function, morphology, and damage in the form of fibrosis detected, visualised, and quantitatively assessed using cardiac MRI. ISO caused changes in cardiac function, morphology, and fibrosis. There was also a differential-distribution of fibrosis-severity across the heart as shown visually by high-resolution sequential MRI images.

\section{PHOSPHODIESTERASE-5 INHIBITION WITH SILDENAFIL SUPPRESSES CALCIUM WAVES BY REDUCING SARCOPLASMIC RETICULUM CONTENT}

David Hutchings*, Katharine Dibb, Charles Pearman, George Madders, Lori Woods, David Eisner, Andrew Trafford. University of Manchester

10.1136/heartjnl-2017-311726.177

Rationale: Occurrence of diastolic $\mathrm{Ca}^{2+}$ waves in cardiac myocytes leads to arrhythmias by inducing delayed after-depolarisations. Waves are initiated when sarcoplasmic reticulum (SR) content reaches a critical threshold level. The phosphodiesterase-5 inhibitor sildenafil (Sil) is antiarrhythmic in mammalian myocardial ischaemia models, while Sil reduces $\mathrm{Ca}^{2+}$ transient amplitude and sarcoplasmic reticulum (SR) $\mathrm{Ca}^{2+}$ content in rat myocytes.

Objective: To determine effects of Sil on propensity to $\mathrm{Ca}^{2+}$ waves in the large mammal.

Methods: Sheep ventricular myocytes were voltage clamped and intracellular $\mathrm{Ca}^{2+}$ measured using Fura-2. Cells were paced at $0.5 \mathrm{~Hz}$ with depolarisations from $-40 \mathrm{mV}$ to $+10 \mathrm{mV}$. When at steady state, waves were induced with 10$15 \mathrm{mM} \mathrm{Ca}^{2+}$. Upon regular waving, Sil $(1 \mu \mathrm{M})$ was applied. To determine threshold SR content, caffeine $(10 \mathrm{mM})$ was added immediately following a wave, and both wave and caffeine-induced $I_{\mathrm{NCX}}$ integrated. Differences between groups were determined using students paired t tests.
Results: Increasing external $\mathrm{Ca}^{2+}$ to $10-15 \mathrm{mM}$ increased SR content and induced diastolic waves. Sildenafil abolished waves in 9/15 cells. In cells where Sil terminated waves, SR content was reduced below threshold. In addition, Sil treatment was associated with a reduced rate constant of SERCA ( $\mathrm{k}_{\text {SERCA }}-66.0 \pm 9.9 \%$ of control, $\mathrm{p}<0.005$ ), an initial (first $4 \mathrm{~s}$ ) increase in sarcolemmal efflux via the $I_{\mathrm{NCX}}$ tail current $(+142$ $\pm 36.4 \%, \mathrm{p}<0.01)$, and reduced sarcolemmal influx via $I_{\mathrm{Ca}-\mathrm{L}}$ $(-30.5 \pm 5.6 \%, \mathrm{p}<0.005)$. In cells continuing to wave in Sil, SR threshold for waves was unchanged $\left(126.9 \mu \mathrm{molL}^{-1}\right.$ ctrl vs 147.2 $\left.\mathrm{molL}^{-1} \mathrm{Sil}, \mathrm{p}=0.6\right)$. In unstimulated cells spontaneously waving in $10-15 \mathrm{mM} \mathrm{Ca}^{2}$, sildenafil reduced wave frequency (6.3 waves per $20 \mathrm{~s}$ vs $2.7, \mathrm{p}<0.005)$. The protective effect of sildenafil on both wave models was abolished when cells were pre-incubated with the PKG inhibitor, KT5823. Sildenafil suppression of waves was also observed in cells from animals in end-stage heart failure, while Sil suppressed ventricular ectopy and episodes of torsades de pointes in vivo in a sheep model of LQT2.

Conclusions: Sildenafil suppresses waves induced by elevated external $\mathrm{Ca}^{2+}$ via a PKG-dependent mechanism, and mediated by a reduction in SR content, which itself is caused by reduced SERCA function \pm reduced $I_{\text {Ca-L }}$. These findings highlight novel antiarrhythmic properties of PDE5 inhibition and translate to suppression of triggered arrhythmias in vivo.

Funding: British Heart Foundation.

\section{THE AORTA CAN ACT AS SITE OF T CELL PRIMING AND PROMOTES A LOCAL CD4+ ADAPTIVE IMMUNE RESPONSE IN EARLY STAGE ATHEROSCLEROSIS IN APOLIPOPROTEIN-E-/- MICE}

Neil MacRitchie*, Gianluca Grassia, Catherine Hughes, Jonathan Noonan, Juliane Schroeder, Paul Garside, Pasquale Maffia. University of Glasgow

10.1136/heartjnl-2017-311726.178

Artery tertiary lymphoid organs (ATLOs) in the adventitia adjacent to intimal plaque in aortas from aged apolipoprotein$\mathrm{E}(\mathrm{apoE})^{-/-}$mice have recently been shown by us to orchestrate the aortic immune response in the advanced stages of experimental atherosclerosis, highlighting the importance of the vascular immune response and its contribution to the pathology. Antigen presenting cells (APCs) and $\mathrm{T}$ cells are found in both human and animal na $\tilde{A} f \hat{A}^{-}$ve and atherosclerotic vessels; however, the mechanisms leading to $\mathrm{T}$ cell activation in the arterial wall remain poorly understood. Here we utilised flow cytometry to present a quantitative assessment of the major antigen presenting cells in both $\mathrm{C} 57 \mathrm{BL} / 6$ wild type (WT) and apoE ${ }^{-/-}$mice in the aorta, aortic draining lymph nodes and spleen. By employing a model antigen and antibody detections system (E $\alpha-G F P / Y-A e)$, it was possible to assess the ability of aortic APC subsets to present antigen in vivo. We also performed a comprehensive phenotypic analysis of $\mathrm{CD}^{+}$ $\mathrm{T}$ cells in healthy versus atherosclerotic aorta using surface marker expression and cytokine signatures. This study revealed that aortas from atherosclerotic mice contained more $\mathrm{CD} 4^{+} \mathrm{T}$ cells and more Th1 T cells (IFN- $\gamma^{3}+$ ) compared to WT, as well as showing a phenotypic switch from a nave to an activated phenotype. On the contrary, changes in $\mathrm{T}$ cell phenotype in the spleen and draining lymph nodes between WT and apoE $^{-/}$mice were either equivalent or modest. One hypothesis regarding the presence of $\mathrm{T}$ cell autoreactive clones 
in the atherosclerotic vessel is that $\mathrm{CD}^{+} \mathrm{T}$ cells are activated locally in the vessel by plaque derived antigens. To determine if $\mathrm{T}$ cell priming can occur locally in the aorta, we first identified nave $\mathrm{T}$ cells in the aorta of WT and OT-II mice (transgenic mice with $\mathrm{CD}^{+} \mathrm{T}$ cells with a TCR specific for chicken ovalbumin 323-339 peptide in the context of $\mathrm{I}_{-} \mathrm{A}^{\mathrm{b}}$ ) and then challenged OT-II mice with ovalbumin while blocking lymphocyte trafficking/egress and extravasation. These experiments revealed that nave $\mathrm{T}$ cells could not only be primed directly in the vessel wall but that the kinetics and respective magnitude of $\mathrm{T}$ cell proliferation to antigen was comparable with aortic draining lymph nodes and spleen, supporting a role for the vessel as a seat of $\mathrm{T}$ cell priming. Together this data illustrates that the aorta can support antigen presentation and that $\mathrm{CD}^{+}{ }^{+} \mathrm{T}$ cells in the atherosclerotic aorta develop a more activated phenotype associated with Th1 cytokine expression, cytokines known for promoting macrophage activation and plaque instability. Most importantly, we demonstrate that the aorta has the capacity to mimic lymphoid tissue in being able to support local antigen driven proliferation of $\mathrm{CD}^{+} \mathrm{T}$ cells.

\section{NOX COMPARTMENTALIZATION AND PROTEIN OXIDATION IN VASCULAR SMOOTH MUSCLE CELLS - IMPLICATIONS IN VASCULAR DYSFUNCTION IN HYPERTENSION}

Livia de Lucca Camargo*, Augusto Cesar Montezano, Sofia Tsiropoulou, Adam Harvey, Katie Hood, Zhenbo Cao, Richard Burchmore, Neil Bulleid, Rhian Touyz. University of Glasgow

\subsection{6/heartjnl-2017-311726.179}

NADPH oxidases (Noxs) are a major source of reactive oxygen species (ROS) in vascular cells. ROS are important signalling molecules with diverse actions. Mechanisms underlying differential ROS effects may relate, in part, to subcellular localization and Nox isoform specificity. We investigated the compartmentalization of Noxs and ROS in vascular smooth muscle cells (VSMC) and questioned whether these phenomena are altered in hypertension. VSMCs isolated from mesenteric arteries of Wistar-Kyoto (WKY) and stroke-prone spontaneously hypertensive rats (SHRSP) were studied. Subcellular compartmentalization of Noxs was evaluated by immunoblotting after organelle fractionation. ROS levels were measured by chemiluminescence $\left(\mathrm{O}_{2}^{-}\right)$and amplex red $\left(\mathrm{H}_{2} \mathrm{O}_{2}\right)$ in the absence or presence of of ML171 (Nox1 inhibitor), GKT136901 (Nox1/4 inhibitor), mito-tempol (mitochondrialtargeted antioxidant) and 4-PBA (ER stress inhibitor). Protein oxidation was assessed using the fluorescent probe DCP-Rho1 for protein sulfenylation and the oxyblot assay for protein carbonylation. Oxidation of protein tyrosine phosphatases (PTP) was evaluated by immunoblotting and Peroxiredoxin (Prx) oxidation was assessed by one-dimensional isoelectric focusing. Vascular reactivity was assessed by myography \pm DTT (reducing agent) and peroxiredoxin inhibitor (Conoidin A). Expression of Nox1, Nox 2 and Nox4 was greater in total cell homogenates from SHRSP versus WKY. Nox isoforms were detected in plasma membrane, ER and nucleus in both strains, but not in the mitochondria. Basal ROS generation was increased in SHRSP cells. In WKY only Nox1 inhibition decreased Ang II-induced ROS generation. Inhibition of Nox1 and Nox4 decreased basal and Ang II-induced ROS in SHRSP.
Additionally, mito-tempol and 4-PBA reduced basal ROS generation in SHRSP. Analysis of protein oxidation revealed increased protein carbonylation and PTP oxidation in SHRSP. Furthermore, oxidation of the antioxidant enzymes Prxs was increased in SHRSP. Prx2, localised in the cytosol, and mitochondrial Prx3 were more oxidised in SHRSP cells than WKY cells. Noradrenaline-induced vascular contraction was reduced by DTT and Conoidin A. Our data demonstrate that Noxs are expressed in an organelle-specific manner, with Nox1,2,4 present in plasma membrane, ER and nucleus, but not in mitochondria. In SHRSP VSMCs Nox expression, ROS generation and protein oxidation are increased. Inhibition of oxidation attenuated vascular reactivity. These findings suggest an important role for Nox1/4 in oxidative stress and post-translational modification of proteins, processes that may play an important role in vascular dysfunction in hypertension.

\section{SYNDECAN-1 IS INVOLVED IN OSTEOPROTEGERIN- INDUCED VASCULAR DYSFUNCTION}

Augusto Montezano*, Rheure A Lopes, Karla Neves, Delyth Graham, Rhian M Touyz. University of Glasgow

\subsection{6/heartjnl-2017-311726.180}

Osteoprotegerin (OPG), an inhibitor of vascular calcification, has pleiotropic vascular effects independently of its actions on calcification. OPG has been associated with vascular inflammation and remodelling and may be important in cardiovascular disease where OPG levels may be elevated. Molecular mechanisms and functional consequences of OPG stimulation in the vasculature are unclear. We propose that syndecan-1, a membrane glycoprotein, may be important and that reactive oxygen species (ROS) play a role in OPG signalling. Vascular reactivity of resistance arteries from WKY rats was studied by wire myography in the presence or absence of OPG $(50 \mathrm{ng} /$ $\mathrm{mL}$ ) and/or synstatin (SSNT $-10^{-6} \mathrm{M}-$ syndecan-1 inhibitor). Rat endothelial cells (EC) and vascular smooth muscle cells (VSMC) were studied. Levels of ROS were measured by chemiluminescence, Amplex Red $\left(\mathrm{H}_{2} \mathrm{O}_{2}\right)$ and ELISA (nitrotyrosine; peroxynitrite - $\mathrm{ONOO}^{-}$). Protein oxidation and levels were measured by immunoblotting. Exposure of resistance arteries to OPG induced endothelial (decreased relaxation to acetylcholine) and VSMC (decreased relaxation to sodium nitroprusside - SNP) dysfunction, as well as, increased contraction to phenylephrine. All responses were blocked by SSNT, N-acetylcisteine (antioxidant) and ML171 (Nox inhibitor). In EC, OPG-induced ROS production (240 $\pm 46.1 \%$ increase $v s$. veh, $\mathrm{p}<0.05)$ was blocked by SSNT. OPG decreased $\mathrm{H}_{2} \mathrm{O}_{2}$ production/release $(61 \pm 5.4 \%$ vs. veh) and increased eNOS Thr 495 phosphorylation (inhibitory site) $(100 \pm 24 \%$ vs. veh, $\mathrm{p}<0.05)$. In VSMC, OPG increased $\mathrm{H}_{2} \mathrm{O}_{2}$ $(69 \pm 3 \%)$ and ONOO $^{-}(43 \pm 12 \%)$ levels, protein oxidation (61 $\pm 15 \%)$, Rho kinase $(200 \pm 39 \%)$ and myosin light chain activation $(55 \pm 3 \%)$ (all $v s$. veh, $\mathrm{p}<0.05)$. Increase in OPGinduced $\mathrm{ONOO}^{-}$levels was exacerbated by SNP $(130 \pm 16 \%$ vs. veh, $\mathrm{p}<0.05)$, a nitric oxide donor. In conclusion, vascular dysfunction elicited by OPG is mediated by syndecan- 1 and ROS. Whether syndecan-1 also impacts on OPG-sensitive calcification is unclear. Our data identify a novel molecular mechanism through syndecan- $1 /$ ROS that may underlie injurious effects of OPG. 